\title{
Xác định các nguồn dị thường từ liền kề bằng phương pháp cực đại wavelet và sự chuẩn hóa tham số tỉ lệ
}

- Dương Quốc Chánh Tín

Trường Đại học Khoa học Tự nhiên, ĐHQG-HCM

- Dương Hiếu Đẩu

- Nguyễn Minh Tân

Trường Đại học Cần Thơ

Email:dqctin@ctu.edu.vn

\section{TÓM TẮT}

(Bài nhận ngày 03 tháng 05 năm 2017, nhận đăng ngày 23 tháng 05 năm 2017)
Trong việc giải bài toán ngược truờng thê, xác định twơng đối chinh xác vị trí các nguồn gây ra dị thuờng tù và trọng lực cùng các thuộc tính của chúng đóng một vai trò rất quan trọng. Với các nguồn dị thuờng tù liền kề, chúng luôn chồng lên nhau không chi trong miền không gian mà còn cả trong miền tần số, gây khó khăn lớn trong việc định vị các nguồn này. Trong bài báo này, nhóm tác giả đã sử dụng một họ wavelet mới để phân tích hiệu quả nhũng thuộc tính của các nguồn truờng thế liền kề. Bằng nhũng mô hình lý thuyết, sư dụng phuoơng pháp cực đại độ lớn biến đổi wavelet, chúng tôi đã xây dưng được hàm tuơng quan giũa tham số tỉ lệ trong phép biến đổi wavelet và độ sâu của nguồn dị thường tù. Hơn thế nữa, sụ chuẩn hóa tham số tỉ lệ cũng được áp dụng để cải thiện độ phân giải, giúp tách biệt các nguồn này trong tỉ lệ đồ, tù đó xác định được độ sâu của chúng. Sau khi kiểm chứng độ tin cậy và tính khả thi của phuơng pháp được đề xuất trên các số liệu mô hình, chúng tôi đã phân tích một số tuyến đo tì tiêu biểu ở đồng bằng Sông Cửu Long. Các kết quả phân tích trong nghiên cứu này là khá phù hợp với các phân tích được công bố truơớc đây, ngoài ra về mức độ chi tiết là khá trùng khớp với các số liệu địa chất khác.

Tù khóa: bài toán ngược truờng thế, nguồn dị thuờng tù̀ liền kề, phuơng pháp cực đại độ lớn biến đổi wavelet, hàm tuoong quan, chuẩn hóa tham số tỉ lẹ

\section{Mở ĐẦU}

Biến đổi wavelet được ứng dụng vào địa vật lý từ đầu thập niên 1980 để phân tích tín hiệu địa chấn [1]. Kể từ đó, những tiến bộ đáng kể của lý thuyết waveletđã mở ra nhiều ứng dụng trong các lĩnh vực khác. Trong vật lý địa cầu, wavelet đã và đang là một công cụ hữu ích trong phân tích các tín hiệu có sự thay đổi đột biến với thời gian [1-4]. Trong lĩnh vực ấy, phân tích dữ liệu trường thế đã có nhiều thành tựu đáng kể khi sử dụng công cụ wavelet để lọc nhiễu, tách trường, xác định vị trí, độ sâu và các đặc tính của nguồn trường đồng nhất [5].

Gần đây, biến đổi wavelet liên tục với hàm wavelet phức Morlet đã được Yang và ccs [6] sử dụng để xác định sự phân bố của các nguồn trường thế. Nhóm nghiên cứu này đã xây dựng được quan hệ xấp xỉ tuyến tính giữa độ sâu của nguồn và số sóng giả (pseudo - wavenumber), để ứng dụng phân tích các số liệu địa từ thực địa. Tuy nhiên, việc chuyển từ miền tham số tỉ lệ sang miền số sóng giả là khá phức tạp và mất nhiều thời gian tính toán, phân tích. Trong bài báo này, qua các mô hình lý thuyết chúng tôi đã xác lập mối tương quan trực tiếp giữa độ sâu của nguồn trường dị thường từ và

Trang 273 
tham số tỉ lệ khi sử dụng phép biến đổi wavelet, để áp dụng vào phân tích một số tuyến đo từ ở vùng Đồng Bằng Sông Cửu Long.

\section{VẠT LIÊU VÀ PHƯƠNG PHÁP} Phép biển đổi wavelet liên tục và hàm phức Farshad - Sailhac

Phép biến đổi wavelet liên tục trên tín hiệu một chiều $\mathrm{f}(\mathrm{x})$ cho bởi:

$W(a, b)=\int_{-\infty}^{+\infty} f(x) \frac{1}{\sqrt{a}} \bar{\psi}\left(\frac{b-x}{a}\right) d x=\frac{1}{\sqrt{a}}\left(f^{*} \bar{\psi}\right)$

với, $a \in \mathrm{R}^{+}$: tham số tỉ lệ và $b \in \mathrm{R}$ : tham số vị trí, $\bar{\psi}(x)$ : liên hiệp phức của $\psi(x)$, là hàm wavelet dùng trong biến đổi, $f * \bar{\psi}$ : ký hiệu tích chập của hàm $f(x)$ và $\bar{\psi}(x)$. Biến đổi wavelet có sự đa dạng khi sử dụng nhiều hàm wavelet chọn lọc khác nhau tùy theo dạng thông tin mà ta phân tích.

Để xác định vị trí theo phương ngang và độ sâu của nguồn dị thường từ, chúng tôi đã sử dụng hàm wavelet phức mới - Farshad - Sailhac [7] có dạng như sau:

$$
\begin{aligned}
& \psi^{(F S)}(x)=\psi^{(F)}(x)+i \psi^{(S)}(x) \\
& \text { trong đó, } \psi^{(F)}(x)=\frac{4-2 x^{2}}{\left(x^{2}+2^{2}\right)^{\frac{5}{2}}}-\frac{1-2 x^{2}}{\left(x^{2}+1^{2}\right)^{\frac{5}{2}}}
\end{aligned}
$$$$
\psi^{(S)}(x)=\operatorname{Hilbert}\left(\psi^{(F)}(x)\right)
$$

Phương pháp cực đại độ lớn biến đổi wavelet (wavelet transform modulus maxima WTMM)

Phương pháp xác định biên theo đề xuất của Mallat và Hwang (1992) [8] liên quan đến việc xây dựng những đường đẳng trị của cực đại độ lớn biến đổi wavelet liên tục trên tín hiệu được phân tích. Điều kiện áp dụng là các hàm wavelet thực thi phải được xác định từ các đạo hàm bậc nhất hay đạo hàm bậc hai của một hàm đặc trưng liên quan đến phép chuyển trường trong bài toán trường thế. Hàm wavelet có tên là Farshad - Sailhac được kiểm chứng là thỏa mãn các yêu cầu của phương pháp Mallat và Hwang, vì thế việc tính toán, phân tích và minh giải vị trí theo phương ngang cũng như độ sâu của các khu vực có dị thường từ mạnh đều dựa trên thành phần độ lớn của biến đổi wavelet này. Kỹ thuật phân tích biên này dựa vào việc xác định vị trí trên tỉ lệ đồ mà tại đó có sự hội tụ của các đường đẳng trị cực đại của độ lớn hệ số biến đổi wavelet nên được gọi là phương pháp cực đại độ lớn biến đổi wavelet (wavelet transform modulus maxima - WTMM).

Trong phương pháp khử nhiễu tín hiệu và tăng độ tương phản cho cách tính biên đa tỉ lệ sử dụng biến đổi wavelet thì Yansun $\mathrm{Xu}$ và ccs [9] có sử dụng cách tính wavelet trên gradient của dữ liệu, phương pháp này làm phát hiện rõ hơn vị trí của các nguồn dị thường nhỏ vì dữ liệu gradient liên quan các biến thiên nhanh của tín hiệu. Vì vậy, trong các phần tiếp theo của bài báo tác giả sẽ áp dụng đổi wavelet trên tín hiệu gradient dị thường từ toàn phần mà lại không áp dụng trên số liệu dị thường từ toàn phần khi phân tích các mô hình lý thuyết cũng như phân tích dữ liệu thực tế.

\section{Xác định chỉ số cấu trúc}

Giả sử $f(x, z=0)$ là trường từ đo trên mặt đất tạo bởi một nguồn từtrườngđồng nhất nằm ở vị trí $x=0$ và độ sâu $z=z_{0}$ dưới mặt đất. Khi thực hiện biến đổi wavelet của $f(x, z=0)$ với các hàm wavelet được xây dựng từ đạo hàm bậc $\gamma$ theo phương ngang của hàm nhân tử trong công thức chuyển trường lên, các hệ số của biến đổi wavelet sẽ tuân theo định luật tỉ lệ kép liên quan đến hai tham số mũ $\gamma$ và $\beta$ cho bởi Sailhac và $\operatorname{CCS}[10]$ :

$$
W_{f(x, z=0)}^{\gamma}(x, a)=\left(\frac{a}{a^{\prime}}\right)^{\gamma}\left(\frac{a^{\prime}+z_{0}}{a+z_{0}}\right)^{-\beta} W_{f(x, z=0)}^{\gamma}\left(x^{\prime}, a^{\prime}\right)
$$

Trong đó: $\mathrm{x}$ và a lần lượt là các tham số vị trí và tỉ lệ; $\beta$ liên quan đến bậc đồng nhất của nguồn từ trường.

Theo Sailhac với các vật thể có từ tính thì mối liên hệ giữa bậc đồng nhất $\beta$, bậc $\gamma$ của đạo hàm và chỉ số cấu trúc $N$ thể hiện tương quan là:

$N=-\beta-\gamma-1$

Với các vị trí đo đạc $\mathrm{x}$ và $\mathrm{x}$ ' khác nhau, mối quan hệ giữa hệ số a và a' là:

\section{Trang 274}


$\frac{a^{\prime}+z_{0}}{x^{\prime}}=\frac{a+z_{0}}{x}=\mathrm{const}$

Trong bài báo này, chỉ số cấu trúc $N$ của nguồn dị thường được xác định bởi hàm wavelet liên tục Farshard-Sailhac. Vì phần thực của wavelet này là $\psi^{(F)}(x)$ trong biểu thức (3) được tạo thành từ đạo hàm bậc 2 theo phương ngang của nhân Farshard [11]:

$$
\theta(x)=\frac{1}{\left(x^{2}+1^{2}\right)^{\frac{1}{2}}}-\frac{1}{\left(x^{2}+2^{2}\right)^{\frac{1}{2}}} \text { nên } \gamma=2 \text { và do đó }
$$

biểu thức (5) được viết lại như sau:

$\left(\frac{1}{a}\right)^{2} W_{f(x, z=0)}^{2}(x, a)\left(a+z_{0}\right)^{-\beta}=\left(\frac{1}{a^{\prime}}\right)^{2} W_{f(x, z=0)}^{2}\left(x^{\prime}, a^{\prime}\right)\left(a^{\prime}+z_{0}\right)^{-\beta}=$ const

Đặt: $W_{f(x, z=0)}^{2}(x, a)=W_{2}(x, a)$ và lấy logarith hai vế của biểu thức (8) sẽ được:

$\log \left(\frac{W_{2}(x, a)}{a^{2}}\right)=\beta \log \left(a+z_{0}\right)+c$

Như vậy, chỉ số cấu trúc $N$ sẽ được xác định từ hệ số góc $\beta$ của đường thẳng:

$$
Y=\beta \cdot X+c
$$

ở đây, $Y=\log \left(\frac{W_{2}(x, a)}{a^{2}}\right)$ và $X=\log \left(a+z_{0}\right)$

Từ việc xác định chỉ số cấu trúc, có thể ước lượng được hình dạng tương đối của nguồn trường (Bảng 2).

\section{Sự chuẩn hóa tham số tỉ lệ}

Trong thực tế, với các nguồn trường thế liền kề, sự chồng chập trường từ liên quan đến nhiều yếu tố khác nhau như: vị trí, độ sâu và kích thước các nguồn thành phần. Trong trường hợp này, cực đại độ lớn của hệ số biến đổi wavelet trong tỉ lệ đồ tạo bởi nguồn dị thường lớn trội hơn hẳn so với các nguồn dị thường nhỏ, làm cho việc xác định các nguồn nhỏ này gặp không ít khó khăn. Để giải quyết vấn đề này, nhóm tác giả đã áp dụng việc điều chỉnh tham số tỉ lệ nhằm rút ngắn khoảng cách về độ lớn của hệ số biến đổi wavelet trong tỉ lệ đồ giữa nguồn dị thường lớn và các nguồn dị thường nhỏ. Từ đó, tạo điều kiện thuận lợi cho việc định vị các nguồn liền kề được dễ dàng hơn, nhất là các nguồn bé.

Để tách các nguồn trường thế liền kề trong tỉ lệ đồ, chúng tôi đã đưa vào phép biến đổi wavelet một chiều trong biểu thức (1) một tham số hiệu chỉnh $a^{-n}$. Khi đó phép biến đổi wavelet một chiều trên tín hiệu $f(x)$ có thể viết lại như sau:

$$
W^{\prime}(a, b)=a^{-n} \int_{-\infty}^{+\infty} f(x) \frac{1}{\sqrt{a}} \bar{\psi}\left(\frac{b-x}{a}\right) d x
$$

Ở đây $n$ là một hằng số dương, và khi $n=0$ thì tham số tỉ lệ không được chuẩn hóa và phương trình (11) trở về phương trình (1). Trong quá trình phân tích một số mô hình dị thường từ đơn giản, chúng tôi nhận thấy với hàm wavelet Farshad Sailhac thì $n$ có thể thay đổi từ 0 đến 1,5 . Khi $n$ tăng thì các hệ số biến đổi wavelet $W^{\prime}(a, b)$ trong biểu thức (11) giảm và khoảng cách về độ lớn của hệ số biến đổi wavelet trong tỉ lệ đồ giữa nguồn dị thường lớn và các nguồn dị thường nhỏ cũng được rút ngắn hơn, nên độ phân giải hình ảnh cũng được cải thiện hơn. Trong bài báo này, nhóm nghiên cứu chọn $n=1,5$ (độ phân giải cao nhất) để phân tích các nguồn trường thế liền kề trong các mô hình lý thuyết cũng như các số liệu thực tế.

\section{Mối quan hệ giữa hệ số tỉ lệ và độ sâu của nguồn dị thường từ}

Trong biến đổi wavelet, tham số tỉ lệ có liên quan đến độ sâu của nguồn gây ra dị thường. Tuy nhiên, hệ số tỉ lệ không phải là độ sâu và cũng không cho ta thông tin trực tiếp về độ sâu. Bằng việc phân tích tỉ lệ đồ qua các mô hình lý thuyết với nguồn trường được tạo ra từ các vật có hình dạng khác nhau, nhóm tác giả đã chỉ ra được tương quan gần như tuyến tính giữa độ sâu của nguồn $z$ và tích số giữa tỉ lệ $a$ với bước đo $\Delta$ qua hệ số tỉ lệ $k$ :

$$
z=k .(a . \Delta)
$$

Hệ số $k$ ở đây phụ thuộc vào chỉ số cấu trúc của nguồn. Tiếp theo, trong phần kết quả nghiên cứu và thảo luận, hệ số $k$ được xác định và ứng dụng để ước lượng độ sâu của các nguồn dị thường trong phân tích các số liệu thực tế.

\section{KẾT QUẢ VÀ THẢO LUẬN}

Trang 275 


\section{Mô hình lý thuyết}

Mô hình 1: Các nguồn dị thường đơn

Trong mô hình này, nguồn từ trường là một quả cầu đồng nhất, bán kính $R=1,0 \mathrm{~km}$. Nguồn bị từ hóa theo phương thẳng đứng với cường độ từ hóa là $M=6 \mathrm{~A} / \mathrm{m}$. Tâm của quả cầu có tọa độ theo phương ngang $x=50 \mathrm{~km}$, và độ sâu $z=3,0 \mathrm{~km}$. Tuyến đo ở mặt đất có chiều dài $100 \mathrm{~km}$ đi qua quả cầu, khoảng cách các điểm đo là $\Delta=0,2 \mathrm{~km}$; như vậy tọa độ các điểm đo lần lượt là: $0 ; 0,2 ; 0,4 ; \ldots 100$ $\mathrm{km}$ (Hình $1 \mathrm{a}$ là đồ thị của trường từ toàn phần, $1 \mathrm{~b}$ là đồ thị của gradient trường từ toàn phần).

Dựa vào kết quả vẽ đẳng trị (Hình $1 \mathrm{C}$ ) có thể dễ dàng xác định tọa độ của điểm cực đại độ lớn biến đổi wavelet (điểm màu trắng nằm giữa đồ thị): $b=250,0 ; a=13,5$. Nhân giá trị của $b$ với bước đo $\Delta=0,2 \mathrm{~km}$ sẽ được vị trí theo phương ngang của tâm nguồn dị thường: $\mathrm{x}=250,0 \times 0,2=50 \mathrm{~km}$. Giá trị này phù hợp với tọa độ thiết kế $x=50 \mathrm{~km}$ của mô hình. Do đó, cực đại độ lớn biến đổi wavelet trên tỉ lệ đồ là thông tin cho phép xác định chính xác vị trí theo phương ngang của nguồn trường.

Giá trị của hệ số tỉ lệ $a=13,5$ có liên quan đến độ sâu của nguồn trường. Để tìm quy luật biến đổi của độ sâu $z$ theo $a$ chúng tôi lần lượt thay đổi $z$

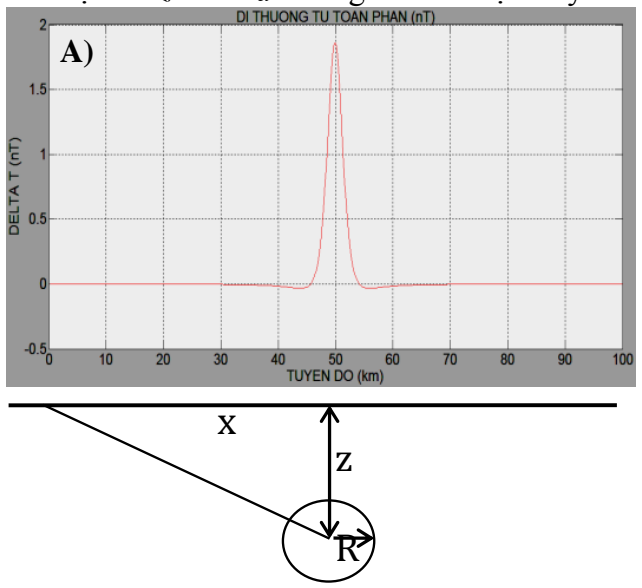

qua các giá trị từ $1,5 \mathrm{~km}$ đến $9,0 \mathrm{~km}$ (bước nhảy $0,5 \mathrm{~km}$ ) và lặp lại quá trình khảo sát như khi $z=3,0$ $\mathrm{km}$. Kết quả khảo sát chỉ ra trong bảng 1 và đồ thị hình 2. Dựa vào đồ thị hình 2 của $z$ theo $a$, xác định được hàm tương quan gần như tuyến tính giữa độ sâu và tham số tỉ lệ là:

$z \approx 1,1247 \times(a . \Delta)(\mathrm{km})$ - khi không chuẩn hóa

$z \approx 4,9918 \times\left(a^{\prime} \Delta\right)(\mathrm{km})$ - sau khi chuẩn hóa với $n=1,5$

Theo Yang và CCS (2010), khi nguồn trường ở xa mặt phẳng đo đạc, chúng thường được giả sử như một khối cầu đồng nhất [6]. Sau đó, độ sâu tương đối của nguồn có thể được ước lượng trực tiếp từ cực đại độ lớn hệ số biến đổi wavelet bởi phương trình (13) - khi không chuẩn hóa tham số tỉ lệ $(n=0)$, hoặc (14) - khi đã chuẩn hóa tham số tỉ lệ $(n=1,5)$.

Trên thực tế các nguồn từ trường có thể có các hình dạng đơn giản khác như: hình trụ, vỉa, đứt gãy hay tiếp xúc. Do đó, cần thiết cho nhóm tác giả tiếp tục thử nghiệm phương pháp của mình với các nguồn từ trường có hình dạng khác. Kết quả tìm hệ số $k$ tương ứng với các nguồn có dạng hình học khác nhau được mô tả ở Bảng 2.
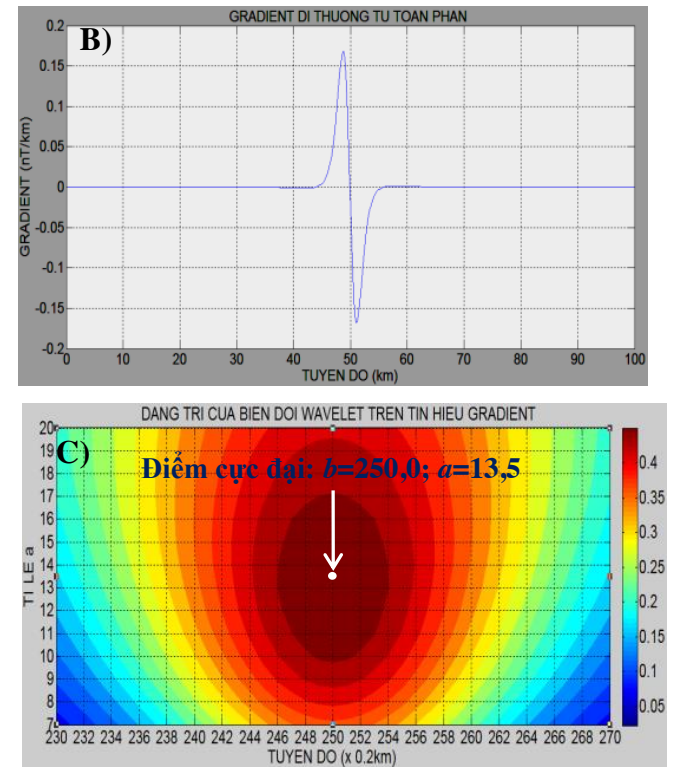

Hình 1. Các dạng đồ thị của mô hình 1 . A) Dị thường từ do một quả cầu đồng nhất gây ra $\mathrm{B})$ Gradient dị thường từ, $\mathrm{C}$ ) Đẳng trị của biến đổi wavelet trên tín hiệu gradient 
TẠP CHÍ PHÁT TRIỂN KHOA HỌC \& CÔNG NGHỆ: CHUYÊN SAN KHOA HỌC TỤ’ NHIÊN, TậP 1, SỐ 6, 2017

Bảng 1. Kết quả phân tích với hàm Farshard - Sailhac

\begin{tabular}{|c|c|c|c|c|c|}
\hline $\mathrm{z}(\mathrm{km})$ & $\Delta(\mathrm{km})$ & $\mathrm{a}(\mathrm{n}=0)$ & $(\mathrm{a} . \Delta)$ & $\mathrm{a}^{\prime}(\mathrm{n}=1,5)$ & $\left(\mathrm{a}^{\prime} . \Delta\right)$ \\
\hline 1,5 & 0,2 & 6,8 & 1,36 & 1,4 & 0,28 \\
\hline 2,0 & 0,2 & 9,1 & 1,82 & 2,0 & 0,40 \\
\hline 2,5 & 0,2 & 11,3 & 2,26 & 2,5 & 0,50 \\
\hline 3,0 & 0,2 & 13,5 & 2,70 & 3,0 & 0,60 \\
\hline 3,5 & 0,2 & 15,8 & 3,16 & 3,6 & 0,72 \\
\hline 4,0 & 0,2 & 17,9 & 3,58 & 4,1 & 0,82 \\
\hline 4,5 & 0,2 & 20,1 & 4,02 & 4,6 & 0,92 \\
\hline 5,0 & 0,2 & 22,4 & 4,48 & 5,0 & 1,00 \\
\hline 5,5 & 0,2 & 24,6 & 4,92 & 5,5 & 1,10 \\
\hline 6,0 & 0,2 & 26,8 & 5,36 & 6,0 & 1,20 \\
\hline 6,5 & 0,2 & 29,1 & 5,82 & 6,6 & 1,32 \\
\hline 7,0 & 0,2 & 31,3 & 6,26 & 7,0 & 1,40 \\
\hline 7,5 & 0,2 & 33,5 & 6,70 & 7,6 & 1,52 \\
\hline 8,0 & 0,2 & 35,8 & 7,16 & 7,9 & 1,58 \\
\hline 8,5 & 0,2 & 38,0 & 7,60 & 8,5 & 1,70 \\
\hline 9,0 & 0,2 & 40,1 & 8,02 & 9,0 & 1,80 \\
\hline
\end{tabular}

Chỉ số cấu trúc $N$ là một thông số giúp xác định hình dạng tương đối của các nguồn trường (từ hay trọng lực trên cột 2 của Bảng 2) là các số nguyên và nó được giới thiệu lần đầu bởi Thompson, D.T., 1982 [12] thông qua phương trình thuần nhất có dạng như sau:

$$
\left(x-x_{0}\right) \frac{\partial T}{\partial x}+\left(y-y_{0}\right) \frac{\partial T}{\partial y}+\left(z-z_{0}\right) \frac{\partial T}{\partial z}=N\left(T_{0}-T\right)
$$

trong đó, $\left(x_{o}, y_{o}, z_{0}\right)$ là vị trí của nguồn dị thường, $T$ là cường độ từ toàn phần đo tại tọa độ $(x, y, z)$, $T_{0}$ là trường từ toàn phần khu vực, $N$ là chỉ số cấu trúc của nguồn dị thường.

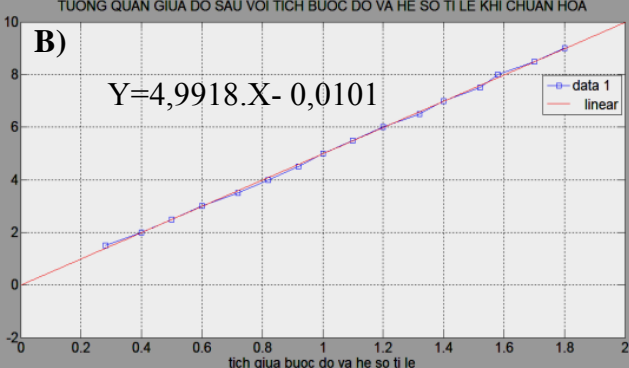

Hình 2. Tương quan giữa độ sâu với tích của bước đo và hệ số tỉ lệ A) Khi chưa chuẩn hóa tham số tỉ lệ; B) Khi đã chuẩn hóa tham số tỉ lệ với $n=1,5$.

Bảng 2. Chỉ số cấu trúc $N$ và tham số $k$ tương ứng

\begin{tabular}{|c|c|c|c|}
\hline Hình dạng & Chỉ số cấu trúc $N$ & $k(n=0)$ & $k^{\prime}(n=1,5)$ \\
\hline Quả cầu & 3 & 1,1247 & 4,9918 \\
\hline Hình trụ & 2 & 1,0991 & 4,4214 \\
\hline Vỉa mỏng & 1 & 0,5981 & 3,6475 \\
\hline Đứt gãy hoặc tiếp xúc & 0 & 0,2026 & 2,0474 \\
\hline
\end{tabular}


Mô hình 2: Nguồn dị thường tì̀ gồm hai quả cầu liền kề

Trong mô hình này, trường từ toàn phần được tạo ra bởi hai quả cầu có kích thước khác nhau. Nguồn bị từ hóa theo phương thẳng đứng như nhau với cường độ từ hóa là $M=6 \mathrm{~A} / \mathrm{m}$. Quả cầu thứ nhất có bán kính $1,0 \mathrm{~km}$ và ở tọa độ theo phương ngang $x_{l}=43 \mathrm{~km}$, và độ sâu $z_{l}=3,0 \mathrm{~km}$; quả cầu thứ hai ở tọa độ theo phương ngang $x_{2}=50 \mathrm{~km}$, và độ sâu $z_{2}=9,0 \mathrm{~km}$ có bán kính $6,0 \mathrm{~km}$. Tuyến đo ở mặt đất có chiều dài $100 \mathrm{~km}$ đi qua hai quả cầu, khoảng cách các điểm đo là $\Delta=0,2 \mathrm{~km}$; như vậy tọa độ các điểm đo lần lượt là: $0 ; 0,2 ; 0,4 ; \ldots 100$ $\mathrm{km}$.

Trong trường hợp hai quả cầu liền kề, nếu chỉ áp dụng phương pháp như trong mô hình 1 thì rất khó xác định được vị trí của quả cầu thứ nhất vì ảnh hưởng rất mạnh của trường từ tạo bởi quả cầu thứ hai (Hình $3 \mathrm{~A}$ và $3 \mathrm{~B}$ lần lượt là đồ thị trường từ và gradient từ toàn phần của mô hình). Thật vậy, quan sát kết quả vẽ đẳng trị trong Hình $3 \mathrm{C}$, chỉ thấy một điểm cực đại của biến đổi wavelet trên tỉ lệ đồ, vị trí điểm này có tọa độ ( $b=252,0 ; a=41,0)$ tương ứng với vị trí nguồn lớn do quả cầu thứ hai tạo ra.
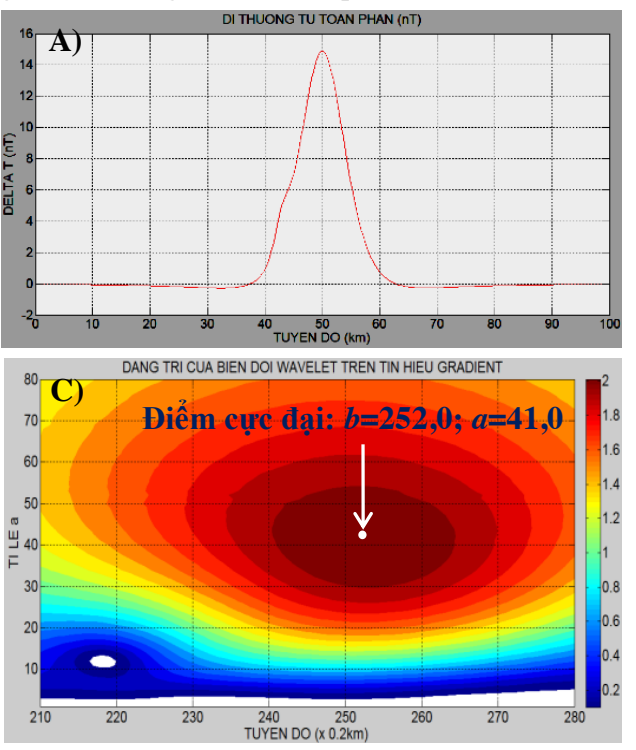

Hình 3. Các dạng đồ thị của mô hình 2. A) Dị thường từ toàn phần do hai quả cầu đồng nhất gây ra, B) Gradient dị thường từ toàn phần, $\mathrm{C}$ ) Đẳng trị của biến đổi wavelet trên gradient từ toàn phần, $\mathrm{D}$ ) Đẳng trị của biến đổi wavelet trên gradient từ toàn phần khi chuẩn hóa
Quả cầu thứ nhất tạo dị thường từ toàn phần khá nhỏ chỉ khoảng $1 / 10$ quả cầu thứ hai, nên hệ số wavelet do nó đóng góp trong tỉ lệ đồ cũng rất nhỏ so với hệ số biến đổi wavelet do quả cầu thứ hai tạo ra tại cùng không gian và do đó, rất khó xác định nguồn thứ nhất trên tỉ lệ đồ.

Để giải quyết vấn đề này, nhóm nghiên cứu đã sử dụng tham số chuẩn hóa $a^{-n}$ (với $n=1,5$ ) trong phương trình (11) trên dữ liệu gradient dị thường từ toàn phần tạo bởi hai quả cầu. Kết quả vẽ đẳng trị được cho bởi hình $3 \mathrm{~d}$ cho thấy tồn tại hai điểm cực đại có tọa độ lần lượt là $\left(b_{1}=218,0\right.$; $\left.a_{1}^{\prime}=3,2\right)$ và $\left(b_{2}=249,0 ; a_{2}^{\prime}=8,8\right)$. Nhân $b_{1}$ rồi $b_{2}$ với bước đo $\Delta=0,2 \mathrm{~km}$ ta được tọa độ theo phương ngang của tâm hai nguồn dị thường: $\mathrm{x}_{1}=2180 \times 0,2=43,6 \mathrm{~km}$ và $\mathrm{x}_{2}=2490 \times 0,2=49,8 \mathrm{~km}$. Nhân $a_{1}^{\prime}$ rồi $a_{2}^{\prime}$ với bước đo $\Delta=0,2 \mathrm{~km}$ và hệ số $\mathrm{k}$ $=4,9918$ (Bảng 2) ta được độ sâu đến tâm của hai nguồn dị thường: $\mathrm{z}_{1}=4,9918 \times(0,3 \times 0,2)=3,2 \mathrm{~km}$ và $\mathrm{z}_{2}=4,9918 \times(8,8 \times 0,2)=8,8 \mathrm{~km}$. Các giá trị này có lệch một ít với các thông số của mô hình do sự tương tác từ giữa hai quả cầu đã làm cho tâm của chúng có xu hướng xích lại gần nhau hơn.
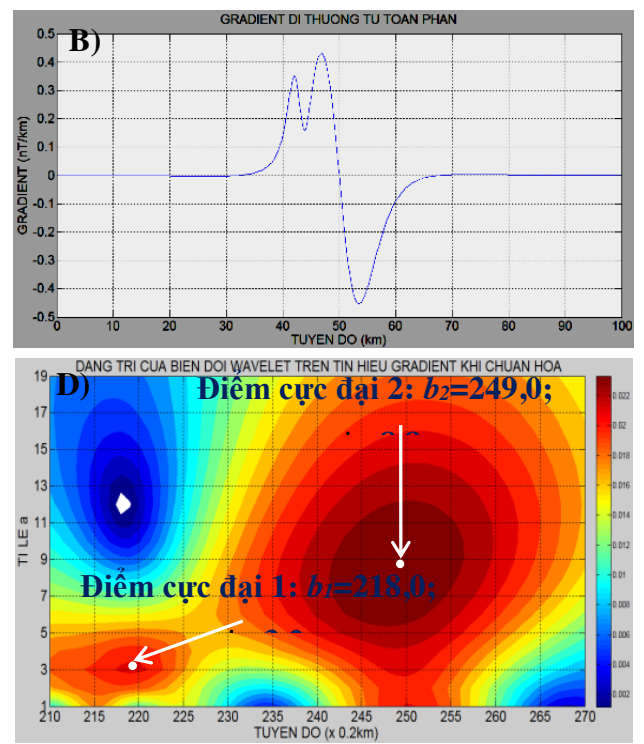

\section{Trang 278}


Nhằm tăng tính thuyết phục của phương pháp được đề xuất, nhóm nghiên cứu tiếp tục phân tích trên các số liệu mô hình được tạo bởi hai nguồn trường từ liền kề có dạng hình học khác nhau gồm một hình trụ nằm ngang và một vỉa mỏng nằm ngang.

Mô hình 3: Nguồn dị thuờng tù gồm một hình trụ nằm ngang đặt liền kề vói một via mỏng nằm ngang

Trong mô hình này, trường từ toàn phần được tạo ra bởi một hình trụ nằm ngang đặt liền kề với một vỉa mỏng nằm ngang. Nguồn cùng bị từ hóa theo phương thẳng đứngvới cường độ từ hóa là $M$ $=6 \mathrm{~A} / \mathrm{m}$. Hình trụ có bán kính $6 \mathrm{~km}$ và ở tọa độ theo phương ngang $x_{1}=44 \mathrm{~km}$, và độ sâu $z_{1}=8,0$ $\mathrm{km}$, trong khi vỉa mỏng ở tọa độ theo phương ngang $x_{2}=50 \mathrm{~km}$, và độ sâu $z_{2}=3,0 \mathrm{~km}$; bề dày 40 $\mathrm{m}$. Tuyến đo ở mặt đất, chiều dài $100 \mathrm{~km}$ đi qua hai nguồn, bước đo là $\Delta=0,2 \mathrm{~km}$ (Hình 4$)$; như vậy tọa độ các điểm đo lần lượt là: $0 ; 0,2 ; 0,4 ; \ldots 100$ $\mathrm{km}$.

Kết quả vẽ đẳng trị (Hình 4c) cho thấy chỉ một điểm cực đại của hệ số wavelet xuất hiện trên tỉ lệ đồ, có tọa độ $(b=222,0 ; a=50,0)$ tương ứng với


tọa độ của hình trụ ngang trong mô hình. Với vỉa ngang, vì dị thường từ toàn phần do nó gây ra không đáng kể so với hình trụ, nên hệ số wavelet do nó đóng góp trong tỉ lệ đồ cũng rất nhỏ so với hệ số biến đổi wavelet do hình trụ ngang tạo ra tại cùng không gian và do đó, rất khó xác định vỉa ngang trên tỉ lệ đồ.

Sử dụng tham số chuẩn hóa $a^{-n}$ (với $n=1,5$ ) trong phương trình (11) trên dữ liệu gradient từ toàn phần tạo bởi hai nguồn. Kết quả vẽ đẳng trị được cho bởi hình $4 \mathrm{~d}$ cho thấy tồn tại hai điểm cực đại có tọa độ lần lượt là $\left(b_{1}=218,0 ; a_{1}^{\prime}=8,8\right)$ và $\left(b_{2}=254,0 ; a_{2}^{\prime}=4,4\right)$. Nhân $b_{1}$ rồi $b_{2}$ với bước đo $\Delta=$ $0,2 \mathrm{~km}$ ta được tọa độ theo phương ngang của tâm hai nguồn dị thường: $\mathrm{x}_{1}=218,0 \times 0,2=43,6 \mathrm{~km}$ và $\mathrm{x}_{2}=254,0 \times 0,2=50,8 \mathrm{~km}$. Nhân $a_{1}^{\prime}$ rồi $a_{2}^{\prime}$ với bước đo $\Delta=0,2 \mathrm{~km}$ và hệ số $k^{\prime}=4,4214$ (tương ứng với hình trụ) hoặc $k^{\prime}=3,6475$ (tương ứng với vỉa mỏng) ta được độ sâu đến tâm của hai nguồn dị thường: $\quad \mathrm{z}_{1}=4,4214 \times(8,8 \times 0,2)=7,8 \quad \mathrm{~km}$ và $\mathrm{z}_{2}=3,6475 \times(4,4 \times 0,2)=3,2 \mathrm{~km}$. Các giá trị này có lệch chút ít với các thông số của mô hình do tương tác từ giữa hai nguồn dị thường.
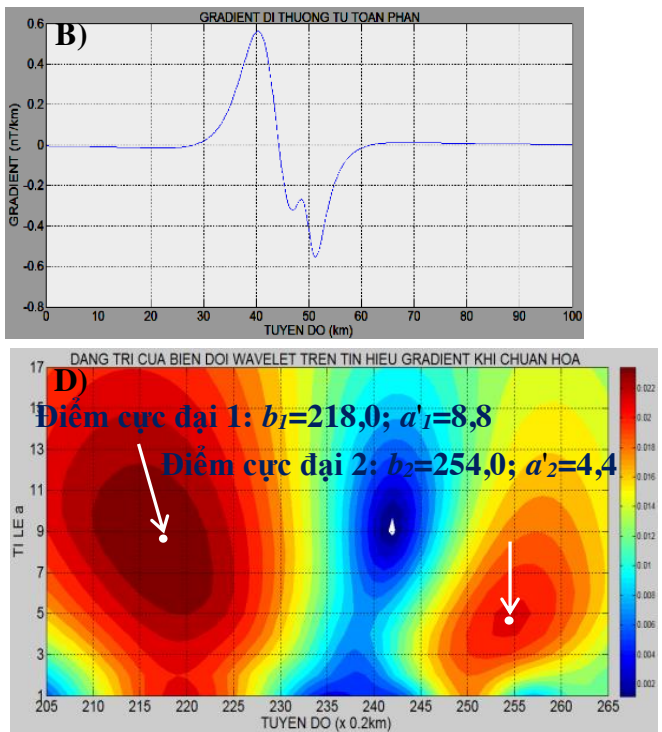

Hình 4. Các dạng đồ thị của mô hình 3. A) Dị thường từ toàn phần do hình trụ ngang và vỉa mỏng ngang gây ra, $\mathrm{B}$ ) Gradient dị thường từ toàn phần, $\mathrm{C}$ ) Đẳng trị của biến đổi wavelet trên gradient từ toàn phần, $\mathrm{D}$ ) Đẳng trị của biến đồi wavelet trên gradient từ toàn phần khi chuẩn hóa 
Tiếp theo, nhóm nghiên cứu tiếp tục thử nghiệm phương pháp trên mô hình có nhiều nguồn dị thường từ hơn, với nhiều hình dạng khác nhau và chúng được bố trí ở các tọa độ cũng rất gần với dữ liệu thực tế nhằm tăng thêm tính thuyết phục về khả năng ứng dụng của phương pháp.

Mô hìn 4: Nguồn dị thường tù gồm một vỉa mỏng nằm gần một quả cầu lớn và một quả cầu nhỏ nằm gần một hình tru lớn.

Trong mô hình này, trường từ toàn phần được tạo ra bởi một vỉa mỏng nằm gần một quả cầu lớn và một quả cầu nhỏ nằm gần một hình trụ lớn. Nguồn cùng bị từ hóa theo phương thẳng đứngvới cường độ từ hóa là $M=6 \mathrm{~A} / \mathrm{m}$. Vỉa mỏng ở tọa độ theo phương ngang $x_{I}=25 \mathrm{~km}$, và độ sâu $z_{I}=1,5$ $\mathrm{km}$; bề dày $40 \mathrm{~m}$; quả cầu lớn có bán kính $3,0 \mathrm{~km}$ và nằm ở tọa độ theo phương ngang $x_{2}=40 \mathrm{~km}$, và độ sâu $z_{2}=4,5 \mathrm{~km}$, trong khi quả cầu nhỏ có bán kính $1,5 \mathrm{~km}$ và nằm ở tọa độ theo phương ngang $x_{3}=80 \mathrm{~km}$, và độ sâu $z_{3}=5,0 \mathrm{~km}$; hình trụ lớn có bán kính $5 \mathrm{~km}$ và ở tọa độ theo phương ngang $x_{4}=90 \mathrm{~km}$, và độ sâu $z_{4}=6,0 \mathrm{~km}$. Tuyến đo ở mặt đất, chiều dài $100 \mathrm{~km}$ đi qua bốn nguồn, bước đo là $\Delta=0,2 \mathrm{~km}$ (Hình 5); như vậy tọa độ các điểm đo lần lượt là: $0 ; 0,2 ; 0,4 ; \ldots 100 \mathrm{~km}$.

Kết quả vẽ đẳng trị (Hình $5 \mathrm{C}$ ) cho thấy chỉ xuất hiện hai điểm cực đại của hệ số wavelet trên tỉ lệ đồ, có tọa độ $\left(b_{2}=200,0 ; a_{2}=19,6\right) ;\left(b_{4}=\right.$ 450,$\left.0 ; a_{4}=25,5\right)$ tương ứng với tọa độ của quả cầu lớn ở $\mathrm{km}$ thứ 40 và hình trụ lớn ở $\mathrm{km}$ thứ 90 trong mô hình. Với vỉa mỏng ở $\mathrm{km}$ thứ 25 , và quả cầu nhỏ ở $\mathrm{km}$ thứ 80 , vì dị thường từ toàn phần do nó gây ra không đáng kể so với hình trụ lớn và quả cầu lớn, nên hệ số wavelet do nó đóng góp trong tỉ lệ đồ cũng rất nhỏ so với hệ số biến đổi wavelet do hình trụ lớn và quả cầu lớn tạo ra tại cùng không gian và do đó, rất khó xác định vỉa mỏng và quả cầu nhỏ trên tỉ lệ đồ.

Sử dụng tham số chuẩn hóa $a^{-n}$ (với $n=$ 1,5) trong phương trình (11) trên dữ liệu gradient từ toàn phần tạo bởi bốn nguồn. Kết quả vẽ đẳng trị được cho bởi Hình $5 \mathrm{D}$ cho thấy tồn tại bốn điểm cực đại có tọa độ lần lượt là $\left(b_{1}=125,0 ; a_{1}^{\prime}=2,0\right)$; $\left(b_{2}=200,0 ; \quad a_{2}^{\prime}=4,7\right) ; \quad\left(b_{3}=405,0 ; \quad a_{3}^{\prime}=5,4\right) ;$ $\left(b_{4}=450,0 ; a_{4}^{\prime}=6,9\right)$. Nhân $b_{1} ; b_{2} ; b_{3}$ rồi $b_{4}$ với bước đo $\Delta=0,2 \mathrm{~km}$ được tọa độ theo phương ngang của tâm bốn nguồn dị thường: $\mathrm{x}_{1}=125,0 \times 0,2=25,0 \mathrm{x}_{2}=200,0 \times 0,2=50,0 \mathrm{~km}$; $\mathrm{x}_{3}=405,0 \times 0,2=81,0 \mathrm{~km}$;và $\mathrm{x}_{4}=450,0 \times 0,2=90,0 \mathrm{~km}$. Nhân $a_{1}^{\prime} ; a_{2}^{\prime} ; a_{3}^{\prime}$ rồi $a_{4}^{\prime}$ với bước đo $\Delta=0,2 \mathrm{~km}$ và hệ số $k^{\prime}=3,6475$ (tương ứng với vỉa mỏng) hoặc $k^{\prime}=4,9918$ (tương ứng với quả cầu) hay $k^{\prime}=$ 4,4214 (tương ứng với hình trụ) ta được độ sâu đến tâm của bốn nguồn dị thường: $\mathrm{z}_{1}=3,6475 \times(0,2 \times 0,2)=1,5 \mathrm{~km}$; $\mathrm{z}_{2}=4,9918 \times(4,7 \times 0,2)=4,7 \mathrm{~km}$; $\mathrm{z}_{3}=4,9918 \times(5,4 \times 0,2)=5,4 \mathrm{~km}$ vàz $z_{4}=4,4214 \times(6,9 \times 0,2)=6,1 \mathrm{~km}$. Các giá trị này có lệch chút ít với các thông số của mô hình do tương tác từ giữa các nguồn dị thường gần nhau.

Trang 280 
TẠP CHÍ PHÁT TRIỂN KHOA HỌC \& CÔNG NGHẸ: CHUYÊN SAN KHOA HỌC TỤ’ NHIÊN, TậP 1, SỐ 6, 2017


Hình 5. Các dạng đồ thị của mô hình 4. A) Dị thường từ toàn phần do vỉa mỏng, hai quả cầu và hình trụ gây ra, B) Gradient dị thường từ toàn phần, $\mathrm{C}$ ) Đẳng trị của biến đổi wavelet trên gradient từ toàn phần,d) Đẳng trị của biến đổi wavelet trên gradient từ toàn phần khi chuẩn hóa

Từ các kết quả khả quan khi phân tích các số liệu mô hình, chúng tôi đã xây dựng một quy trình xác định tọa độ và độ sâu của các nguồn dị thường từ liền kề để áp dụng phân tích các tuyến đo thực tế.

Quy trình xác định tọa độ và độ sâu các nguồn từ liền kề bằng phép biến đổi wavelet Farshard - Sailhac

Việc xác định tọa độ và độ sâu của nguồn từ liền kề sử dụng biến đổi wavelet Farshard -
Sailhac có thể tóm lược trong quy trình gồm các bước sau:

Bước 1: Lấy gradient ngang của trường từ toàn phần dọc theo tuyến đo.

Bước 2: Thực hiện biến đổi wavelet trên gradient ngang của trường từ bằng hàm wavelet Farshard - Sailhac.

Sau biến đổi wavelet liên tục phức, thu được bốn bộ số liệu khác nhau gồm: phần thực, phần 
phức, phần độ lớn, và phần pha. Dữ liệu của phần độ lớn sẽ được sử dụng trong các bước kế tiếp.

Bước 3: Thay đổi hệ số tỉ lệ $a$ và lặp lại biến đổi wavelet Farshard - Sailhac đa tỉ lệ.

Bước 4: Vẽ đẳng trị của hệ số biến đổi wavelet Farshard - Sailhac trên gradient ngang trong tỉ lệ đồ $(a, b)$.

Bước 5: Xác định tọa độ theo phương ngang của nguồn dị thường.

Trên đồ thị đẳng trị, xác định các điểm cực đại của hệ số wavelet. Tọa độ theo phương ngang và phương thẳng đứng lần lược là $b_{i}$ và $a_{i},(i$ chỉ số thứ tự của nguồn). Tọa độ theo phương ngang của các nguồn gây ra dị thường từ được xác định bởi biểu thức sau:

$$
x_{i}=b_{i} \times \Delta
$$

Bước 6: Xác định độ sâu của nguồn trường.

Tính chỉ số cấu trúc của các nguồn đã xác định trong bước 5 , từ đó ước lượng hình dạng tương đối của nguồn, rồi xác định hệ số $k_{i}$ hoặc $k_{i}^{\prime}$ tương ứng từ bảng 2 . Khi đó, độ sâu của các nguồn từ được xác định bởi biểu thức sau:

$$
\begin{aligned}
& z_{i}=k_{i} \cdot\left(a_{i} \cdot \Delta\right) \\
& z_{i}=k_{i}^{\prime} \cdot\left(a_{i}^{\prime} \cdot \Delta\right)
\end{aligned}
$$

Phương trình (17) áp dụng khi không chuẩn hóa tham số tỉ lệ ( $n=0)$, và phương trình (18) áp dụng khi đã chuẩn hóa tham số tỉ lệ $(n=1,5)$.

\section{Phân tích tuyến đo từ ở vùng đồng bằng Nam bộ}

Áp dụng quy trình trên để xác định tọa độ và độ sâu của nguồn từ với hàm wavelet Farshard Sailhac trên các số liệu thực tế, chúng tôi đã phân tích sáu tuyến đo từ trên bản đồ cường độ từ toàn phần ở Đồng bằng Sông Cửu Long. Các kết quả phân tích đều cho thấy độ chính xác khá tốt, phù hợp với các công bố của các tài liệu địa chất trước đây. Tuy nhiên, trong bài báo này, nhóm chỉ trình bày kết quả phân tích tuyến Cà Mau - Sóc Trăng.

Chúng tôi sử dụng bản đồ cường độ từ toàn phần với tỉ lệ $1 / 500.000$ được cung cấp bởi Cục địa chất và khoáng sản Việt Nam. Chúng tôi chọn tuyến đo chạy dọc từ Cà Mau đến Sóc Trăng, độ dài $103 \mathrm{~km}$, sau đó dữ liệu được nội suy với khoảng cách mỗi điểm đo cách đều nhau $\Delta=1 \mathrm{~km}$. Sử dụng trường từ trung bình tham chiếu quốc tế của Đại học Kyoto, nhóm tác giả đã tính được cường độ dị thường từ toàn phần của tuyến đo. Kết quả được mô tả trên hình $5 \mathrm{a}$, qua đồ thị phát hiện hai dị thường mạnh ở gần vị trí $\mathrm{km}$ thứ $42 ; 89$ và hai dị thường yếu hơn ở $\mathrm{km}$ thứ 27 và 80 . Các dị thường này có cả phần dị thường dương và dị thường âm kề nhau. Cực đại dị thường có giá trị khoảng $150 \mathrm{nT}$ ở $\mathrm{km}$ thứ 8 và cực tiểu của dị thường có giá trị là $-230 \mathrm{nT}$ ở km thứ 89 .

Dựa vào kết quả vẽ đẳng trị trên hình $6 \mathrm{c}$, có thể xác định được dọc theo tuyến đo có hai nguồn gây ra dị thường mạnh tương ứng với hai điểm cực đại độ lớn biến đổi wavelet: $b_{1}=42, a_{1}=4,0 ; b_{2}=$ $89, a_{2}=5,5$. Trong đó, nguồn dị thường thứ hai có quy mô và cường độ lớn hơn hẳn nguồn dị thường thứ nhất.

Lấy các giá trị $b_{1} ; b_{2}$ nhân với bước đo $\Delta=1$ $\mathrm{km}$ ta được tọa độ của các nguồn gây ra dị thường từ dọc theo tuyến đo tương ứng tại các $\mathrm{km}$ thứ 42 và 89.

Để xác định tọa độ hai nguồn dị thường nhỏ ở $\mathrm{km}$ thứ 27 (gần nguồn dị thường mạnh hơn ở km thứ 42) và $\mathrm{km}$ thứ 80 (gần nguồn dị thường rất mạnh ở km thứ 89) nhóm nghiên cứu đã sử dụng tham số chuẩn hóa $a^{-n}$ (với $n=1,5$ ) trong biến đổi wavelet cho bởi phương trình (11) trên dữ liệu gradient dị thường từ toàn phần dọc theo tuyến đo. Kết quả vẽ đẳng trị (hình $6 \mathrm{~d}$ ) cho thấy xuất hiện thêm hai vị trí cực đại độ lớn của hệ số wavelet: $b_{3}=27, a_{3}^{\prime}=0,44 ; b_{4}=80, a_{4}^{\prime}=0,86$. Lấy các giá trị $b_{3} ; b_{4}$ nhân với bước đo $\Delta=1 \mathrm{~km}$ được tọa độ của hai nguồn gây ra dị thường từ nhỏ dọc theo tuyến đo tương ứng với các $\mathrm{km}$ thứ 27 và 80 .

Tiếp theo, để xác định độ sâu của các nguồn dị thường này, chúng tôi bắt đầu với đường biểu diễn $\log \left(W / a_{i}^{2}\right)$ theo $\log \left(a_{i}+z\right)$ nhằm tính chỉ số cấu trúc.

\section{Trang 282}


TẠP CHÍ PHÁT TRIỂN KHOA HỌC \& CÔNG NGHỆ: CHUYÊN SAN KHOA HỌC TỰ NHIÊN, TẬP 1, SỐ 6, 2017
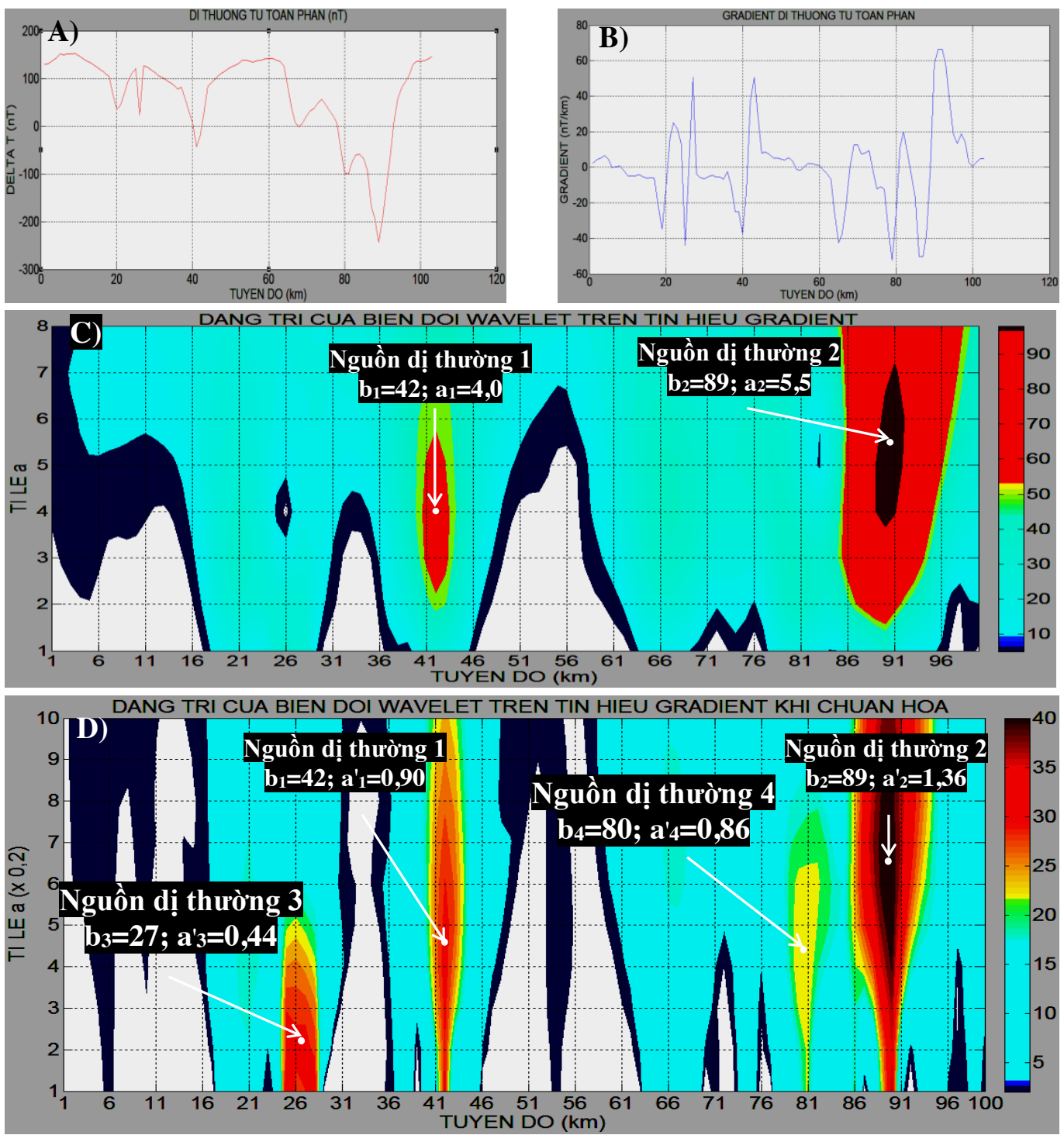

Hình 6. Các dạng đồ thị của tuyến đo thực tế. A) Dị thường từ trên tuyến đo, B) Gradient dị thường từ, C) Đẳng trị của biến đối wavelet trên gradient dị thường từ,

D) Đẳng trị của biến đổi wavelet trên gradient dị thường từ khi đã chuẩn hóa

Hình 7D vẽ đường biểu diễn của $\log \left(W / a_{i}^{2}\right)$ theo $\log \left(a_{i}+z\right)$ khi phân tích dữ liệu của nguồn dị thường ở $\mathrm{km}$ thứ 89 trên tuyến đo. Sử dụng phương pháp bình phương tối thiểu, phương trình đường thẳng: $Y=-5,1 X+11,9$ đã được xác định, sau đó chúng tôi ước lượng giá trị của $\beta \approx-5$ (biểu thức 10), do đó chỉ số cấu trúc là $N=5-2-1=2$ (phương trình 6 ). Như vậy nguồn dị thường từ này có dạng tương đối là hình trụ, tương ứng với hệ số tỉ lệ $k=1,0991$ hoặc $k^{\prime}=4,4214$ (Bảng 2). Nhân hệ số tỉ lệ $k$ với $\left(a_{2} . \Delta\right)$ hoặc $k^{\prime}$ với $\left(a_{2}^{\prime} . \Delta\right)$ ta được độ sâu của nguồn trường ở km thứ 89 khoảng 6,0 $\mathrm{km}$. Phân tích tương tự cho các dị thường còn lại trên tuyến đo, thu được kết quả tổng hợp trong Bảng 3. 

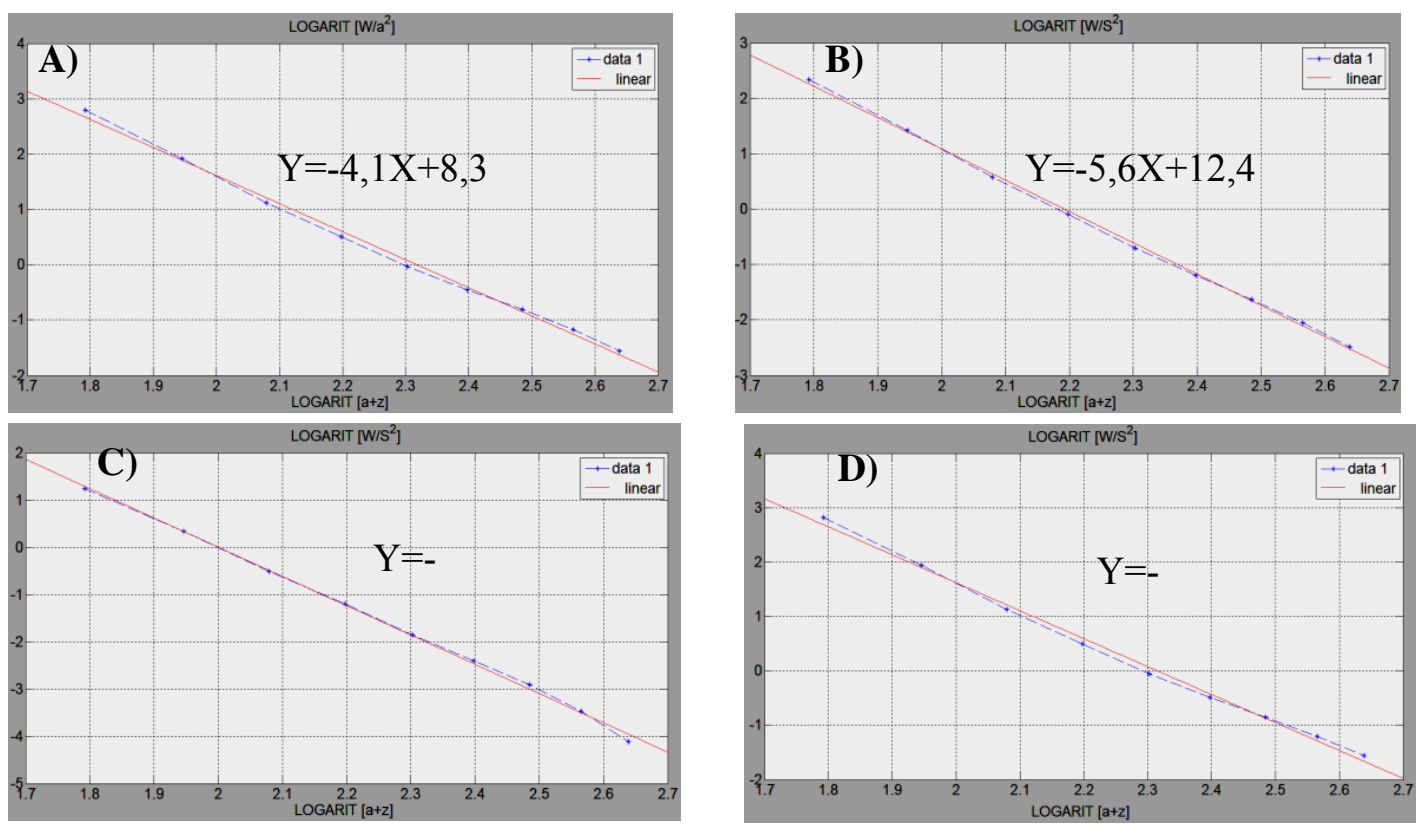

Hình 7. Các đồ thị biểu diễn đường $\log \left(W / a_{i}^{2}\right)$ theo $\log \left(a_{i}+z\right)$. A) Nguồn dị thường ở km thứ 27 ,

B) Nguồn dị thường ở km thứ 42, C) Nguồn dị thường ở km thứ $80, \mathrm{D})$ Nguồn dị thường ở km thứ 89

Bảng 3. Tổng hợp kết quả phân tích các nguồn dị thường từ trên tuyến đo Cà Mau - Sóc Trăng

\begin{tabular}{|c|c|c|c|c|c|}
\hline TT & $\begin{array}{c}\text { Vị trí ngang } \\
(\mathrm{km})\end{array}$ & $\begin{array}{c}\text { Bậc đồng nhất } \\
\beta\end{array}$ & $\begin{array}{c}\text { Chỉ số cấu trúc } \\
\mathrm{N}\end{array}$ & $\begin{array}{c}\text { Hình dạng } \\
\text { tương đối }\end{array}$ & $\begin{array}{c}\text { Độ sâu } \\
(\mathrm{km})\end{array}$ \\
\hline 1 & 42 & 6 & 3 & Cầu & 4,5 \\
\hline 2 & 89 & 5 & 2 & Trụ & 6,0 \\
\hline 3 & 27 & 4 & 1 & Vỉa & 1,6 \\
\hline 4 & 80 & 6 & 3 & Cầu & 4,3 \\
\hline
\end{tabular}

\section{KẾT LUẬN}

Chúng tôi đã sử dụng một họ wavelet mới có tên là Farshard - Sailhac để giải bài toán ngược trường thế nhằm xác định tọa độ, độ sâu và chỉ số cấu trúc của các nguồn gây ra dị thường từ liền kề. Qua việc phân tích các mô hình lý thuyết, sử dụng phương pháp cực đại độ lớn biến đổi wavelet, nhóm tác giả đã thiết lập được hàm tương quan gần như tuyến tính giữa độ sâu với hệ số tỉ lệ. Việc chuẩn hóa tham số tỉ lệ cũng được áp dụng để cải thiện độ phân giải, giúp tách biệt các nguồn dị thường liền kề trong tỉ lệ đồ, từ đó xác định được độ sâu của chúng. Quy trình xác định tọa độ và độ sâu của nguồn dị thường từ bằng hàm wavelet Farshard - Sailhac đã được xây dựng và áp dụng. Kết quả phân tích tuyến đo từ Cà Mau - Sóc Trăng cho thấy có bốn nguồn gây ra dị thường từ. Trong đó, về quy mô thì có hai nguồn lớn gây ra dị thường mạnh và hai nguồn bé gây ra dị thường yếu hơn. Về hình dạng thì có hai nguồn dạng cầu, một nguồn dạng trụ, và một nguồn dạng vỉa với tọa độ, độ sâu và chỉ số cấu trúc của chúng là khá trùng khớp với các công bố trước đó [13].

\section{Trang 284}




\section{Identification of magnetic anomalies of adjacent sourses using the wavelet transform modulus maxima and scale normalization}

- Duong Quoc Chanh Tin

University of Science, VNU-HCM

- Duong Hieu Dau

- Nguyen Minh Tan

Cần ThơUniversity

ABSTRACT

In the potential field inverse problems, accurate determination of the location for the anomaly sources and their properties played an important role. For geomagnetic anomalies of adjacent sources, they always superimpose upon each other not only in the spatial domain but also in the frequency domain, making the identification of these sources significantly problematic. In this paper, a new mother wavelet for effective analysis the properties of the close potential field sources was used. By theoretical modeling, using the wavelet transform modulus maxima (WTMM) method, we set up a correlative function between the scale parameter and geomagnetic source depth. Moreover, a scale normalization on the wavelet coefficients was introduced to enhancethe resolution for the separation of these sources in the scalograms, thereby determining their depth. After verifying the reliability of the proposed method on the modeling data, we have analysed the geomagnetic data in the Mekong delta. The results of this interpretation were consistency with previously published ones, furthermore, the level of resolution for this technique was quite coincidental with other methods using different geological data.

Keywords: potential field inverse problems, geomagnetic anomalies of adjacent sources, the wavelet transform modulus maxima (WTMM) method, correlative function, scale normalization

\section{TÀI LIỆ THAM KHẢO}

[1]. P. Kumar, E. Foufoula-Georgiou, Wavelet analysis for geophysical applications, Reviews of Geophysics, 35, 4, 385-412 (1997).

[2]. S. Ouadfeul, Automatic lithofacies segmentation using the wavelet transform modulus maxima lines (WTMM) combined with the detrended fluctuation analysis (DFA), $17^{\text {th }}$ International geophysical congress and exhibition of Turkey, Expanded abstract (2006).

[3]. S. Ouadfeul, Very fines layers delimitation using the wavelet transform modulus maxima lines WTMM combined with the
DWT, SEG SRW, Expanded abstract, (2007).

[4]. S. Ouadfeul, L. Aliouane, S. Eladj, Multiscale analysis of geomagnetic data using the continuous wavelet transform, Application to Hoggar (Algeria), SEG Expanded, Abstracts 29, 1222; doi:10.1190/1.3513065 (2010).

[5]. M. Fedi, T. Quarta, Wavelet analysis for the regional - residual separation of potential field anomalies, Geophysical Prospecting, 46, 507-525 (1998).

[6]. Y. Yang, Y. Li, T. Liu, Continuous wavelet transform, theoretical aspects and 
application to aeromagnetic data at the Huanghua Depression, Dagang Oilfield, China. Geophysical Prospecting, 58, 669684, European Association of Geoscinetists \& Engineers (2010).

[7]. D.Q.C. Tin., D.H. Dau., Interpretation of the geomagnetic anomaly sources in the Mekong Delta using the wavelet transform modulus maxima, Workshop on Capacity Building on Geophysical Technology in Mineral Exploration and Assessment on Land, Sea and Island, Ha Noi, 121-128 (2016).

[8]. S. Mallat, W.L. Hwang, Singularity Detection and Processing with Wavelets, IEEE Transactions on Information Theory, 38, 2, 617-643 (1992).

[9]. Y. Xu, J.B. Weaver, D.M. Healy Jr., J. Lu., Wavelet transform domain filters: a spatially selective noise filtration technique, IEEE Transactions on Image Processing, 3, 6, 747-758 (1994).
[10]. P. Sailhac, A. Galdeano, D. Gibert, F. Moreau, C. Delor, Identification of sources of potential fields with the continuous wavelet transform: Complex wavelets and applications to magnetic profiles in French Guiana, Journal of Geophysic. Research, 105, 19455-19475 (2000).

[11]. S. Farshard, R.K. Amin, H.R. SiahKoohi, Interpretation of 2-D Gravity Data using 2D Continuous Wavelet Transform Introduction, $72^{\text {nd }} E A G E$ Conference \& Exhibition incorporating SPE EUROPEC, Barcelona, Spain (2010).

[12]. D.T. Thompson, EULDPH: A new technique for making computer-assisted depth estimates from magnetic data, Geophysics, 47, 31-37 (1982).

[13]. Dương Hiếu Đẩu, Phân tích tài liệu tù và trọng lục sử dụng biến đổi wavelet liên tục, NXB ĐHQG TPHCM (2013). 